\title{
Modelling of a zoonotic pathogen (Campylobactor) in a dairy herd
}

\author{
$\underline{\text { A. Parshotam }}^{\text {a }}$ \\ ${ }^{a}$ National Institute of Water and Atmospheric Research (NIWA), Hamilton, New Zealand \\ Email: a.parshotam@niwa.co.nz
}

\begin{abstract}
Zoonotic pathogens such as Campylobactor are a major contributor of human disease notifications in countries with agricultural economies, such as New Zealand. Understanding the mechanisms by which Campylobactor persist in animal reservoirs is key to designing effective intervention strategies. A model of Campylobacter transmission within a dairy herd was developed based on a Susceptible-InfectedRecovered (SIR) modelling framework. The model incorporates stages of disease progression and includes a compartment (W) that tracks Campylobacter concentration in the environment. The model was used to perform simulations but is yet to be tested against observed data. Threshold parameters for invasion such as the basic reproduction number, $R_{0}$ were estimated to be about 0.19 , indicating that one infectious individual would on average infect 0.19 susceptible animals and the disease would thus not be able to establish itself. The variation of short term dynamics with epidemiological parameters was examined. The model has been developed with a long-term view of understanding and incorporating seasonal and climate change factors. Methods for incorporating these factors into the model are discussed.
\end{abstract}

Keywords: Campylobacter, SIR models, SIWR models 


\section{INTRODUCTION}

Zoonotic pathogens such as Campylobactor are a major contributor of human disease notifications in countries with agricultural economies, such as New Zealand. Epidemiological models based upon the Susceptible-Infected-Recovered (SIR) framework offers useful insights into these diseases and their transmission in human populations. McBride and French (2006) developed a linear model of a human population exposed to Campylobacter, through both food and environmental routes, based on the SIR framework. The model accounted for age-dependency and differential immunity within groups of people. McBride (pers comm.) extended this work to include the effects of climate change on reported human campylobacteriosis rates. Tien and Earn (2010) extended the classical SIR framework by adding a compartment (W) that tracks Campylobacter concentration in the water environment. Invasion threshold parameters were computed from the resulting SIWR model.

Zoonotic pathogens (e.g., E. coli, Campylobacter, oocysts of Cryptosporidium, Salmonella) are commonly found in cattle. Understanding the mechanisms by which these pathogens persist in animal reservoirs is vital to the design of effective intervention strategies. Epidemiological models based upon the SIR framework have provided useful insights into infectious diseases and their transmission over farmed landscapes. Xiao et al. (2005) developed an SIR mathematical model of the dynamics of Salmonella infections in dairy herds. Multiple groups (unweaned, weaned, dry and lactating) of animals were considered. Matthews et al. (2008) developed a model that captured within-herd transmission dynamics of toxic E. coli O157, herd-to-herd movement of infected animals and reservoirs of infections. This information was used to determine thresholds for persistence of these $E$. coli in a metapopulation of herds. They found that equilibrium prevalences in the Scottish national herd were sensitive to key parameters - herd-to-herd movement rates, group size and the within-group reproduction ratio/number, $R_{0}$. Chapagain et al. (2008) developed a mathematical model of the transmission dynamics of Salmonella to describe an outbreak that occurred in a Pennsylvania dairy herd. Multiple stages of infection were considered and $R_{0}$ was calculated and related to intervention strategies. Marshall and French (2010) developed a model of Campylobacter carriage and transmission between and within animal groups. Estimates of direct and indirect transmission based on a simple deterministic single-group model were extended to a multi-group model by apportioning the indirect rates between the local (such as in water troughs, pasture, or on equipment) and general environments based on how much between-group transmission was expected to occur. Seasonality due to maturation of the animals, which is dependent on date of birth of the animal and various maturation ages were incorporated into their model.

The objective of this paper is to develop a model of Campylobacter transmission within a dairy herd. The model incorporates $n$ stages of disease progression and includes a compartment (W) that tracks Campylobacter concentration in the environment. The model is an extension of the classical SIR model and is referred to by Tien and Earn (2010) as part of a family of SI ${ }^{\mathrm{n}} \mathrm{WR}$ models. The model was used to perform simulations and is yet to be tested against observed data. The basic reproduction number, $R_{0}$ is calculated. The variation of short term dynamics with epidemiological parameters and the variation of $R_{0}$ are examined. Parameters sensitive to climate change are discussed and methods for deriving expressions for timedependent threshold conditions, $R_{0}$ are discussed with a view to extending the model to understand and incorporate seasonal variation and climate change factors into future models.

\section{THE MODEL}

The model used was a state-transition model adopted from Chapagain et al. (2008) and Xiao et al. (2005) for modelling Salmonella in dairy herds. It has been possible to adopt this model for modelling Campylobactor because the transmission parameters are not given in any explicit form. Note that there is much work done in New Zealand on Campylobactor in the environment and in animals. There is however, not much Salmonella in cattle in New Zealand largely because animals feed on grass in New Zealand and Salmonella is often spread with contaminated animal food.

The model is an extension of the SIR model given in Lloyd (2001), for $n$ stages of the disease with changing infectivity. This model arises out of replacing the infectious period distribution from a usual exponential distribution to a gamma distribution.

The animals are grouped into three compartments according to their Campylobacter infection status. In the presence of the disease the total population of the herd $(N)$ is composed of three population classes: susceptibles (denoted by $S$ ), infected (denoted by $I$ ) and immunes/recovered (denoted by $R$ ). The infected animals progress through several $(n)$ stages of the disease. Indirect transmission due to free-living bacteria in the environment is modelled by including the density of pathogen in the environment $(W)$ and is a function of 
the total number of infected animals shedding the bacteria and the bacteria survival rate in the environment. For convenience, a latent period after infection is not considered in the model. Figure 1 shows a flow diagram for the transmission dynamics described by the resulting SI ${ }^{\mathrm{n}} \mathrm{WR}$ model system, where the rate coefficients $\mathrm{n} \gamma$ result from a gamma distribution assumed for the infectious period.

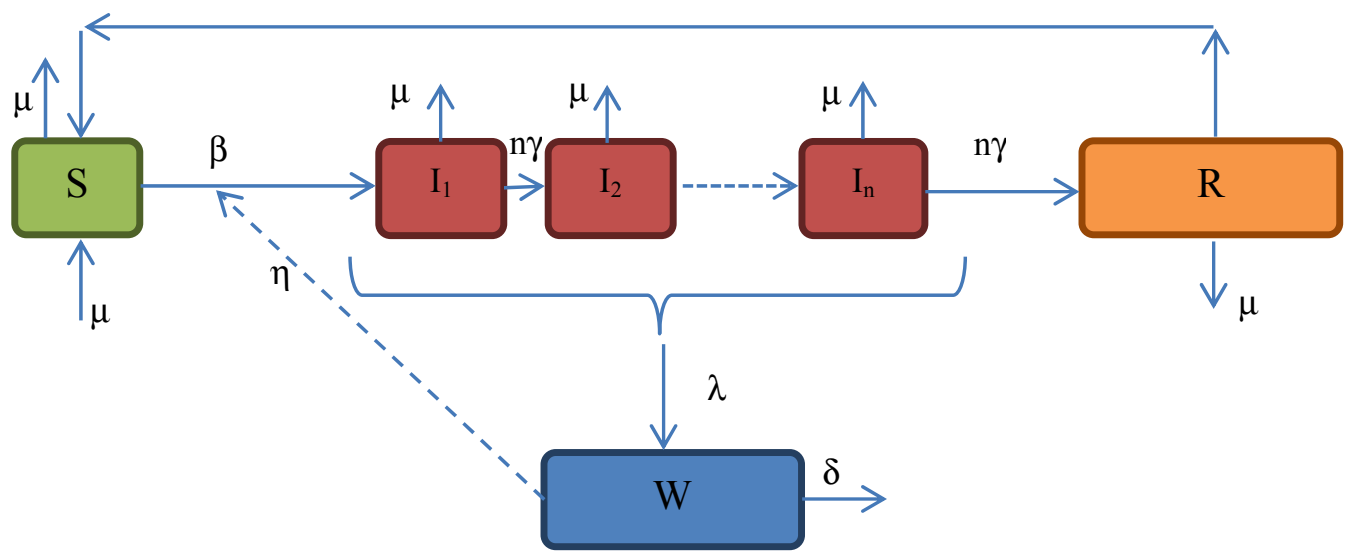

Figure 1. The $\mathrm{SI}^{\mathrm{n}} \mathrm{WR}$ modelling structure representing the transmission dynamics of Campylobacter in a dairy herd modelled by the system of equations (1)-(5). Green box $=$ susceptibles $(S)$. Red box $=$ infectious $(I)$; Orange box $=$ immune/recovered $(R)$; Blue box $=$ Environment $(W)$.

The dynamics of host and pathogen incorporating multiple stages is given by the model:

$$
\begin{aligned}
& \frac{d S}{d t}=\mu N-\frac{(\beta I+\eta \eta W) 5}{N}-\mu S+\phi R \\
& \frac{d I_{1}}{d t}=\frac{(\beta I+\eta W) S}{N}-(n \gamma+\mu) I_{1} \\
& \left(d I_{4} n\right) / d t=n \gamma I_{1}(n-1)-(n \gamma+\mu) I_{1} n\left(n^{\prime \prime} 22\right)= \\
& \frac{d R}{d t}=n \gamma I_{n}-(\phi+\mu) R \\
& \frac{d W^{*}}{d t}=\lambda I-\partial W
\end{aligned}
$$$$
I=\sum_{k=1}^{n} l_{k}
$$

$I$ is the total number of infective animals in various infectious stages, $\sum_{k=1}^{1}$. The rates of transition between various compartments and model parameters (including definitions) are given in Table 1. Parameter estimates are from local New Zealand data, when available and from the literature.
}

Table 1: Definition and parameter estimates for the model that describes a single-infectious stage model of Campylobacter in a dairy herd. The rate coefficients are given in units of per day.

\begin{tabular}{l|lll}
\hline Parameter & Symbol & Value & Sources/remarks \\
\hline Direct transmission parameter & $\beta$ & 0.004 & Marshall and French (2010) \\
Birth and death rate coefficient & $\mu$ & 0.0004 & Marshall and French (2010) \\
Indirect transmission parameter & $\eta$ & $1.3 \times 10^{-}$ & $\begin{array}{l}\text { Unknown. Assumed from Xiao et al. (2008) for } \\
\text { Salmonella }\end{array}$ \\
Rate coefficient of recovery from the infection & $\gamma$ & 0.05 & Marshall and French (2010) \\
Rate coefficient of loss of immunity once infected & $\phi$ & 0.007 & $\begin{array}{l}\text { Unknown. Assumed from Chapagain et al. (2008) for } \\
\text { Ralmonella }\end{array}$ \\
Rate coefficient of addition of pathogen to the & $\lambda$ & $5.8 \times 10^{7}$ & Marshall and French (2010)
\end{tabular}


environment due to shedding by infected animals

Rate coefficient of pathogen removal from the $\delta$ environment
0.14 Marshall and French (2010)

Methods for estimating some of these parameters are given in Chapagain et al. (2008). Transition rates may be estimated from faecal culture data. The distribution of the infectious period is fit with a gamma distribution in order to calculate the average infectious period, $1 / \gamma$ and variance of the distribution, $1 /\left(\mathrm{n} \gamma^{2}\right)$, where $\gamma$ is the rate at which an infected individual recovers from the infection. These two quantities give the number of stages that the single infectious compartment needs to be divided in the model described by the set of equations (1)-(5). The direct transmission parameter, $\beta$ is estimated from the number of infectious animals and total number of animals at a given time, and the force of infection, which gives the number of new infections in the sampling interval.

Using the given parameters, the simulation was started at a time $t=0$ with a set of initial conditions. The population size, $N$ assumed is 100 and considered large enough. This is the size of a very small herd of animals. Note that because the model is deterministic and continuous, population size matters a lot. The consequences of small fluctuations are far bigger in smaller populations and in dealing with large populations; chance events will be averaged out before it effects determines any qualitative change in the system. Initially it was assumed that there are 50 susceptible individuals, zero recovered individuals, zero pathogen in the environment and 25 individuals in infectious stages one and two.

The basic reproduction number, $R_{0}$ (the spectral radius of the next generation matrix) is often used to assess the effect of various control strategies on the persistence of infection (Driessche and Watmough, 2002). The control strategies that reduce $R_{0}$ below one are successful in eliminating the disease. $R_{0}$ is influenced by the epidemiological factors and the variation in $R_{0}$ with some of these factors needs to be considered. For the above system of equations, $R_{0}$ is given by the following equation, derived by Chapagain et al. (2008):

$$
\overline{\left[1-\left(\frac{m}{m+n}\right)^{n}\right]}
$$

The system of nonlinear differential equations (1) - (5) was solved using a fourth-order Runge-Kutta method in the freeware package $\mathrm{R}$ developed by the R Development Core Team (2008). The numerical results were used to observe and quantify effects of epidemiological factors on the short-term behaviour of the system and on the basic reproduction number, $R_{0}$. For a single infectious compartment, $R_{0}>1$ when $\beta>(\gamma+\mu-\lambda \eta / \delta)$. Note that $R_{0}$ in equation (6) is independent of $\phi$, the rate of loss of immunity once infected.

The model was used to perform simulations and has not yet been tested against observed data.

\section{RESULTS}

\subsection{Numerical Simulations}

Using the given parameters, the simulation was started at a time $t=0$ when there were initially 50 susceptible individuals, zero recovered individuals, zero in the environment and 25 individuals in infectious stages one and two $(n=2)$. Figure 2 shows the typical behaviour of the numerical solutions observed.

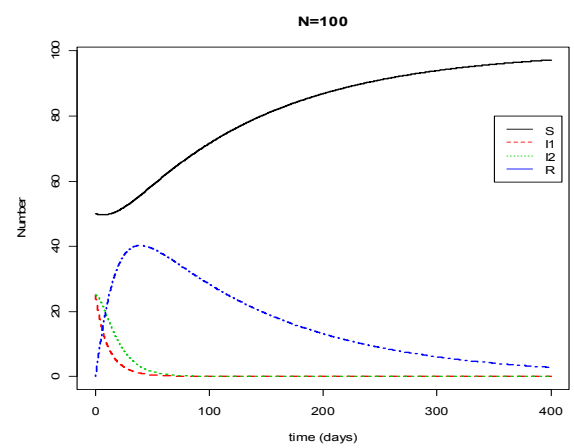

Figure 2. Simulations of susceptible individuals, $S(t)$, infected individuals, Recovered individuals, $R(t)$ in two infectious stages $I_{1}(t)$ and $I_{2}(t)$, by time, $t$ from to $t=0$. The parameter values are given in Table 1 . 


\subsection{Variation in short term dynamics with epidemiological parameters}

Figure 3 presents the dynamic behaviour of the model equations by varying the direct transmission parameter, $\beta$, the rate coefficient of recovery from infection, $\gamma$, and the rate coefficient of loss of immunity once infected, $\phi$. All other parameters are fixed and given in Table 1. The results are given in terms of prevalence of infection (\%) in a population $N$ of 100. Again, there were initially, 50 susceptible individuals, zero recovered individuals and zero in the environment and 25 individuals in infectious stages one and two $(n=2)$. Figure 2 shows the typical behaviour of the numerical solutions observed.
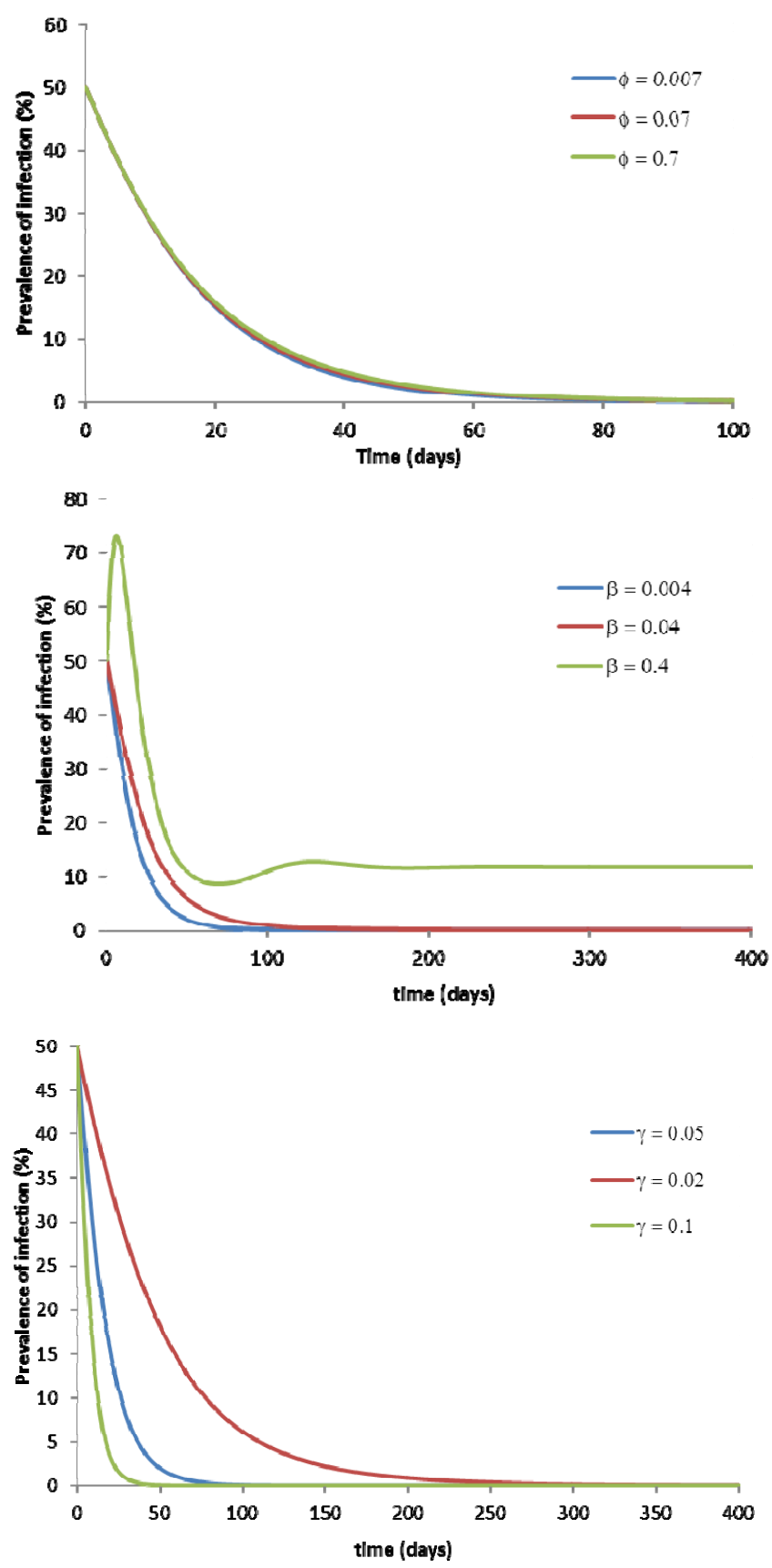

Figure 3. The dynamic behaviour of the model equations by varying the direct transmission parameter, $\beta$, the rate coefficient of recovery from infection, $\gamma$, and the rate coefficient of loss of immunity once infected, $\phi$. These are given in terms of prevalence of infection (\%) in a population $N$ of 100 . 


\subsection{Threshold parameters for invasion}

The basic reproduction number, $R_{0}$ is estimated from equation (6) to be 0.19 for $n=2$. It is evident that $R_{0}$ increases with an increase in the number of infectious stages but not by very much with the given set of parameters assumed. The eventual estimate of $R_{0}$ is about 0.2 , indicating that one infectious individual would on average infect 0.2 susceptible animals. With this value of $R_{0}$, the disease cannot have sustained transmission in the population and will become extinct.

\section{DISCUSSION}

It is evident from the derived basic reproduction number, $R_{0}$ of about 0.2 , that the dairy herds are not vulnerable to Campylobacter infection and persistence of the infection. There has been a paucity of rate coefficient and transmission parameter estimates in this study, some data obtained from Salmonella modelling. Better estimates of these parameters are required to refine the model and provide better estimates of $R_{0}$. The model has been given a sensitivity analysis but has not been used to investigate a range of management and control scenarios. Several theoretical results have been obtained in conjunction with collecting further measurement data. These theoretical results are described below.

Climate change can contribute significantly to the transmission of zoonotic diseases in livestock and SIR model parameters as yet have not been 'indexed' to include these factors. Gale et al. (2009) assessed the effect of climate change on livestock diseases by identifying the main factors through which changing climatic conditions affect the biology, transmission and epidemiology of zoonotic and other pathogens. Environmental conditions such as temperature, humidity and sunlight also affect the survival of zoonotic pathogens that are able to survive outside the host in the environment. Increased flood events and increased wetter conditions after flood events can provide a transfer mechanism both within and between farms to contaminate pasture and other areas where livestock have access and can lead to disease outbreaks.

The algebraic expression of the basic reproduction number of the $\mathrm{SI}^{\mathrm{n}} \mathrm{WR}$ gives a synthesis of all epidemic parameters in the model. It is possible to determine the influence of these parameters on the basic reproduction number and this allows one to drive control measures to reduce the basic reproduction number.

The direct transmission parameter $\beta$, as with other model parameters and rates are likely to be time dependent due to seasonal variations. These seasonal effects may give rise to temporal oscillations in the disease prevalence in the herd and can be incorporated into the model. Following Dietz (1976), the transmission rate, $\beta$ can be assumed to be seasonally varying in time (with period 1 year), and a sinusoidal form is used to model it, e.g. $\beta=\beta_{0}(1+\varphi \cos 2 \pi t)$, where $\varphi$ is referred to as the degree of seasonality and $0 \leq \varphi \leq 1$. By substituting $\beta$ into equations (1) and (2), a periodically forced nonlinear system is obtained. It can easily be verified that all state variables in the new system remain non-negative. The behaviour of the SIWR system in the current study can be investigated with respect to the two parameters $\varphi$ and $\beta_{0}$ and a parametric portrait of the model presented to determine parameter sensitivities (see Yu et al. (1994) where this approach has been used for SIR models). Lloyd (2001) has discussed seasonal forcing for a similar SIR model with a number of infectious stages.

Accurate estimates of the seasonal effects in the current study are not generally available but some estimates showing the variation of the transmission parameter $\beta$ throughout the year for Salmonella are given in Chapagain et al. (2008). A time-dependent threshold condition, $R_{0}(t)$ is obtained for dengue fever epidemics by taking an intuitive approach when the transmission components and other model parameters are seasonally dependent and incorporate climate change effects (e.g. Coutinho et al. 2006). The sensitivity of $R_{0}$ with respect to abiotic conditions such as temperature and humidity may be determined explicitly using these results (Massad et al. 2011) and it may be possible to obtain time-dependent threshold conditions for the resulting seasonally forced $\mathrm{SI}^{\mathrm{n}} \mathrm{WR}$ model in the current study using some of these approaches.

\section{ACKNOWLEDGMENTS}

The author thanks Graham McBride for his comments and help in preparing this manuscript.

\section{REFERENCES}

Chapagain, P.P., J.S. van Kessel, J.S. Karns, D.R. Wolfgang, E. Hovingh, K.A. Nelen, Y.H. Schukken, and Y.T. Grohn (2008). A mathematical model of the dynamics of Salmonella Cerro infection in a US dairy herd. Epidemiology and Infection. 136(2): 263-272. 
Coutinho, F.A.B., M.N. Burattini, L.F. Lopez, and E. Massad (2006). Threshold Conditions for a NonAutonomous Epidemic System Describing the Population of Dengue. Bulletin of Mathematical Biology. 68: 2263-2282.

Dietz, K. 1976. The incidence of infectious diseases under the influence of seasonal fluctuations. In: Berger, J. et al. (eds.) Mathematical models in medicine (Lect. Notes Biomath. Vol. 11, 1-15). Springer, Berlin, Heidelberg, New York.

Driessche, P. van den and J. Watmough (2002). Reproduction numbers and sub-threshold endemic equilibria for compartmental models of disease transmission. Mathematical Biosciences. 180: 29-48.

Gale, P., L.P. Drew, G.D. Phillips, and M. Woolridge (2009). The effect of climate change on the occurrence and prevalence of livestock diseases in Great Britain: a review. Journal of Applied Microbiology. 106: $1409-1423$.

Lloyd, A.L. (2001). Realistic distributions of infectious periods in epidemic models: Changing patterns of persistence and dynamics. Theoretical Population Biology 60: 59-71.

Massad, E., F.A.B. Coutinho, L.F. Lopez, and D.R. da Silva (2011). Modelling the impact of global warming on vector-borne infections. Physics of Life Reviews 8: 169-199.

McBride, G. and N. French (2006). Accounting for age-dependent susceptibility and occupation-dependent immune status: A new linear analytical SIR model. WSEAS Transactions on Mathematics. 11(5): 12411246.

Marshall, J. and N. French (2010). Modelling of Campylobacter carriage and transmission between and within animal groups. Report prepared for the New Zealand Food Safety Authority and Ministry for the Environment, Technical Report, Massey University.

Matthews, L., J.C. Low, D.J. Mellor, M.C. Pearce, G.T. Innocent, G.J. Gunn, and W.J. Reid (2008). A multiscale model of E. Coli $\mathrm{O} 157$ transmission in the Scottish cattle population. Society for Veterinary Epidemiology and Preventive Medicine (Eds E.J. Peeler, L. Alban, A. Russell and the SVEPM Executive Committee).

R Development Core Team. 2005. R: A language and environment for statistical computing. R Foundation for Statistical Computing, Vienna, Austria. ISBN 3-900051-07-0, URL http://www.R-project.org.

Tien, J.H. and D.J.D. Earn (2010). Multiple transmission pathways and disease dynamics in a waterborne pathogen model. Bulletin of Mathematical Biology 72: 1506-1533.

Xiao, Y., R.G. Bowers, D. Clancy, and N.P. French (2005). Understanding the dynamics of Salmonella infections in dairy herds: a modeling approach. Journal of Theoretical Biology 233: 159-175.

Yu, A. Kuznetsov and C. Piccardi (1994). Bifurcation analysis of periodic SEIR and SIR epidemic models. Journal of Mathematical Biology 32: 109-121. 